\title{
Les papyrus arabes de Heidelberg disparus. Essai de reconstruction et d' analyse
}

\author{
R.G. Khoury
}

\section{La collection de papyrus arabes de Heidelberg}

La collection de papyrus arabes de Heidelberg est une des plus prestigieuses dans le monde. Non qu' elle soit une des plus importantes concernant le nombre de ses pièces, car elle est nettement inférieure à la plupart de ce que l'on trouve, surtout à Vienne, où les papyrus arabes, et ce que l' on peut rattacher à la papyrologie arabe sont plus de $5 \mathbf{0 . 0 0 0}$ pièces complètes ou fragmentaires.

Entre-temps les fonds de cette collection sont bien connus, pour avoir été décrits, en fonction de beaucoup de publications de certains de ses papyrus. Avant mes travaux concernant les manuscrits historiques rares, et les plus vieux de leur genre, que j' ai publiés au cours des années, et dès $1972^{1}$, Richard Seider en avait livré un aperçu général en 1964 dans la collection «Heidelberger Jahrbücher. $»^{2}$ Notons qu'il s'agit à Heidelberg d'une riche collection, dans laquelle les papyri gréco-latins sont de loin les plus nombreux. A côté de papyrus importants en copte, nous avons des papyri arabes qui ont été acquis par l' Université de Heidelberg, pour être conservés dans sa Bibliothèque Universitaire, vers la fin du $19^{\mathrm{e}}$ et surtout vers le début du $20^{\mathrm{e}}$ siècle. Elle porte comme sigle les lettres PSR, c' est-à-dire Papyri Schott - Reinhardt; ce dernier était un bavarois et avait travaillé des années comme drogman au consulat allemand au Caire, avec une formation générale d' orientaliste pour l'époque; F. Schott était un industriel, originaire de la région de Stuttgart (Göppingen) et était devenu le directeur général de la fabrique de ciment (Portland - Zementwerke, Heidelberg et Mannheim, aujourd' hui seulement Zement AG, Heidelberg), qui est devenue une des meilleures à travers le monde. Ce directeur, un riche mécène, fit don de plusieurs centaines (plus de 1000) pièces de papyrus (et de documents sur papier aussi) à l'Université. La collection resta à l'intérieur de la Bibliothèque Universitaire, jusqu' au moment où elle fut levée en 1976 au rang

1 Là-dessus, voyez Khoury, Wahb ibn Munabbih et Khoury, 'Abd Allāh.

2 Seider, Aus der Arbeit $142 \mathrm{ff}$.

(C) R.G. KHOURY, 2015 | DOI:10.1163/9789004284340_014

This is an open access chapter distributed under the terms of the prevailing CC-BY-NC License at the time of publication. 
d'un Institut de ma Faculté, et cet Institut de Papyrologie a toujours été dirigé par un spécialiste des papyrus grecs, du fait que ceux-ci sont de loin les plus nombreux.

Dans la masse de ces manuscrits il s'agit de documents, de lettres et de contrats de toutes sortes, de mandats d' impôts etc. Loin de vouloir minimiser la valeur de ce genre de pièces, il faut noter que peu d'entre elles sont complètes, ou révolutionnaires dans leur contenu, du fait que nous avons des milliers de documents importants, même très importants, surtout à Vienne, dans la Bibliothèque Nationale Autrichienne (Österreichische Nationalbibliothek), et que d'autres collections à travers le monde occidental, et bien sûr dans la Bibliothèque Nationale (Dār al-kutub) du Caire en ont aussi. Parmi toutes ces pièces, les manuscrits sur pauyrus l'emportent, numériquement.

\section{Quelques raretés spécialement précieuses}

Quelques papyrus sont cependant d'une importance capitale et ont toujours contribué à donner à toute la collection arabe une renommée spéciale, aussi bien en Europe qu' en Orient:

II $/ 1$

Plusieurs lettres de Qurra ibn Sharīk (m. 96 / 714), gouverneur omeyyade d'Egypte, sur des questions administratives, fiscales et économiques, concernant des enquêtes, ou sur des réclamations de rapports sur des faits délictueux à cet égard. Carl Heinrich Becker était le premier à éditer cette collection de lettres, qui est la plus importance numériquement à Heidelberg, en $1906^{3}$.

Naturellement Heidelberg n' est pas le seul lieu, dans lequel sont conservées quelques autres lettres, aussi bien en Europe (Paris, Londres, Vienne) qu'en Egypte (le Caire). Notre collègue Jaser Abu Safiyya vient de donner une édition assez complète de toutes ces lettres, avec une étude générale sur le gouverneur et son activité4.

II $/ 2$

Deux textes historiques admirables sont à nommer, à côté de ces lettres, à l' aide desquelles on peut écrire une véritable grammaire de l'ancien arabe, en

3 P.Heid.Arab. I. Voyez aussi Rāgib, Lettres nouvelles 173-187; Sijpesteijn, Une nouvelle; Dietrich, Die arabischen et Diem, Philologisches.

4 Abū Safiyya 1425/2004. 
complément des textes coraniques. Ils sont attribués à Wahb ibn Munabbih (34-110 ou 114 / 654-655-728 ou 732):

II $/ 2.1$

Hadīth Dāwūd - ou Histoire du roi David, la plus ancienne de son genre dans l'histoire islamique, et qui porte la date la plus vieille qu' un manuscrit arabe littéraire (dans le sens qu'il n' appartient pas au genre des documents purs) ait jamais porté dans la culture arabe: Dhū l-qa'da 229 / juillet 844 . Dès mon édition de ce papyrus et de celui qui suit j'ai insisté plus d'une fois sur le fait qu'il s'agissait là d'une version, à partir d'un original, qui a été exécutée en Egypte, à Fusțāț, dans la maison du juge de ce pays 'Abd Allāh ibn Lahī'a, dont il sera question plus loin, dans la présentation du troisième papyrus historico-littéraire, que j' ai publié avec les autres, dans une série de volumes entre 1972 et 1986.

On y distingue deux parties déterminées:

a. La première concerne le règne de Saül. Il commence par la désignation de ce dernier par le prophète Samuel, à laquelle suit l'histoire de son choix, comme premier roi des Israélites. Puis viennent l'épisode sur l' arche d' alliance (al-Tābūt), le combat de David contre Goliath (Jālūt) et enfin les péripéties autour de la jalousie de Saül vis-à-vis de David, auquel il a donné sa propre fille en mariage, sans pourtant l' associer à son règne, comme il le lui avait promis. Cette partie se termine par la tentative du roi d' assassiner son rival David, par son repentir et par sa mort.

b. La deuxième décrit le règne de David. Elle commence par des détails sur sa manière de vivre et de régner; puis viennent les descriptions de sa tentation, suivie du conte des «plaideurs $»^{5}$, en guise d'introduction aux très belles pages sur le repentir de David, pour avoir convoité et épousé la femme de son officier 'Uriyya, après avoir envoyé ce dernier de campagne en campagne contre les ennemis jusqu'à sa mort; puis une description de la révolte de son fils Absalon contre lui et la mort de celui-ci. Le tout prend fin par les très belles pages sur Salomon et ses trois jugements: la femme convoitée par le juge, le chef de police et le responsable du marché; les deux femmes, les deux enfants et le loup, ainsi que le texte relatif à la Sourate 21, 78-79, auxquels succèdent de brefs récits, entre autres sur la construction du temple, commencée par David, ainsi que sur la mort de celui-ci.

5 Coran 38:21ff. 
II $/ 2.2$

Maghāzì rasūl allāh (terme valable aussi bien pour la vie (Sīra) que pour les véritables campagnes militaires, auxquelles il fut plus tard réservé) ${ }^{6}$.

A l' encontre du papyrus précédent, celui-ci ne porte ni titre, ni date, mais est de la même écriture et appartient, sans doute à la même époque, de laquelle sont certains transmetteurs de tous ces écrits, qui avaient affaire, en Egypte, avec la maison du juge Ibn Lahī‘a, dont il sera tout de suite question. Il comprend les événements suivants de la vie et des campagnes du Prophète Mahomet:

- La rencontre d'al-'Aqaba.

- Le conseil des qurayshites à Dār al-nadwa (Maison du conseil).

- L'émigration de la Mecque à Médine (Hijra).

- La campagne de 'Alī contre la tribu Khath`am et la conversion de celle - ci à l'Islam.

Pour toutes les questions concernant ces textes, leur auteur, ou premier transmetteur, ainsi que leur attribution à lui, c' est-à-dire à Wahb Ibn Munabbih, voyez là-dessus mon livre sur lui ${ }^{7}$.

II $/ 3$

Un dernier texte littéraire est enfin le fameux rouleau de papyrus (șaḥifa) attribué à 'Abd Allāh Ibn Lahī‘a (97-174 / 715-790). Celui-ci était devenu d'abord juge d'Egypte, et puis sa grande autorité, qui avait dans sa maison une bibliothèque, dans laquelle il rassemblait, ou copiait ou laissait copier des originaux ou des copies d'originaux, de sorte que cette bibliothèque fut à l'origine non seulement des manuscrits sur papyrus, décrits ici, mais aussi d'autres qui ont été découverts avec eux et qui formaient des documents de toutes sortes; sans que l'on puisse dire toujours de quels documents exactement il est question, car ils ne portent pas de signes attestant leur provenance, ou la place de leur conservation. Mais du fait que sa bibliothèque était devenue centrale pour l'Egypte de son temps, et aussi pour tous ceux d'entre les savants qui la visitaient, il est évident qu' elle a dû contenir un nombre importants d'autres manuscrits, parmi ses uṣul et furu', dont il est toujours question. Depuis mon livre sur lui, et l'édition de son rouleau, on ne peut plus en douter, d' autant plus

6 Voyez M. Hinds, 'al-Maghāzī,' Encyclopaedia of Islam. New Edition, 5:1161.

7 Khoury, Wahb ibn Munabbih $9 \mathrm{ff}$; $34 \mathrm{ff}$. (hadīth Dāwūd); $118 \mathrm{ff.}$ (Maghāzī), comme auteur de ces papyrus, $183 \mathrm{ff}$. 
que plusieurs de mes travaux qui ont suivi, depuis 1986, ne font que corroborer ces données sur lui ${ }^{8}$.

Ce côté-là est important, pour expliquer la provenance et le lieu de conservation de beaucoup de documents arabes sur papyrus surtout, qui nous sont venus des trois premiers siècles, et qui ont dû avoir comme possesseur, quand on peut les dater, exactement ou approximativement, quand ils ne le sont pas du tout, ou Ibn Lahīa ou quelqu' un qui a travaillé sous son autorité, copié et transmis des manuscrits, sous sa dictée, enseigné comme maitre à son tour et donné le droit de copier, de transmettre et d'enseigner ${ }^{9}$. C'était le plan de Becker lui-même qui prévoyait la publication des papyrus d'Ibn Munabbih et d'Ibn Lahī'a, malheureusement rien n'en a été; et ce que Gertrud Mélamède a essayé de donner s'est limité aux deux premiers feuillets des Maghāzì de Wahb, desquels elle a carrément laissé tomber toute la première page (sauf l' isnād de deux lignes), de la deuxième page manquent cinq lignes, et de la quatrième manquent quatre lignes vers la fin; de plus elle a laissé beaucoup de lacunes etc ${ }^{10}$. Parmi les documents, en dehors de ces textes, dits historiques et littéraires, il y a un certain nombre d'autres pièces, qui ont été publiées par quelques spécialistes, dont je nommerai quelques-uns, à cause de leur importance scientifique (et aussi numérique):

\section{Les documents médicaux}

Plusieurs papyrus ont en effet un contenu médical, et Becker, ayant quitté Heidelberg pour Hambourg, encouragea E. Seidel à se vouer à la publication de

8 Sur lui, v. Khoury, 'Abd Allāh vie et œuvre, $7 \mathrm{ff}$; ; sur sa maison et sa bibliothèque, $27 \mathrm{ff}$; édition, $243 \mathrm{ff}$., $31-32$ où ce passage avait été déjà présenté et traduit par moi, pour la première fois; je l'ai repris plusieurs fois plus tard dans une série de différents articles, dont le dernier est le suivant: L'apport spécialement important de la papyrologie dans la transmission et la codification des plus anciennes versions des Mille et Une Nuits et d' autres livres des deux premiers siècles islamiques, in: Sijpesteijn et Sundelin, Papyrology $70 \mathrm{ff}$. (l' article: 63-95). De plus, al-Jābirī, Takwīn 61 ff., ce livre ne m'était pas à cette époque disponible; j' en ai pris connaissance à travers la critique adressée à lui par Georges Tarābīshī, Ishkāliyyāt $11 \mathrm{ff}$.

9 Concernant ces «Certificats», délivrés à la fin de véritables séances, et appelés Ijāzāt $s a m \bar{a}$, ou "Certificats d'audition et de transmission», qui ont pris place tôt dans la culture de l' Islam classique, voyez Vajda, Les certificats; Sellheim, Gelehrte 54-79; Mackay, Certificates 197; Khoury, Asad ibn Mūsā 91-108, il s' agit là de 28 certificats.

10 Là-dessus, Khoury, Wahb ibn Munabbi 4-5; Mélamède, The meetings 17-58. 
ces documents; celui-ci suivit le conseil et les livra en quatre parties à Becker pour sa revue Der Islam, que celui - ci venait de fonder en 1910:

«Medizinisches aus der Heidelberger Papyri Schott-Reinhardt », I. Teil: Der Islam, 1 (1910): 145-152. II. Teil: Der Islam, 1 (1910): 238-268. III. Teil: Der Islam, 2 (1911): 220-230. IV. Teil: Der Islam, 3 (1912): 273-291.

\section{Le reste des documents travaillés}

Puis 12 ans passèrent, jusqu'à ce que Grohmann publia 11 textes de protocoles, et 10 ans après suivirent 11 textes de magie, travaillés par Grohmann, Bilabel et $\mathrm{Graf}^{11}$. Voilà quelques spécimens, auxquels il faut ajouter quelques textes, surtout des lettres importantes que K. Jahn édita peu après ${ }^{12}$ et un document intéressant, contenant un achat de drogues, qui fut publié par A. Dietrich ${ }^{13}$. Le reste est plus proche de nous, et il s'agit de quelques autres documents ou lettres parus dans différentes publications dans ma Chrestomathie ou dans certains travaux de W. Diem, sur lesquels je ne m'attarde pas ici.

\section{Les papyrus disparus de la collection}

Entre-temps plusieurs membres d'universités surtout arabes ont passé par Heidelberg, ont essayé de classer, d'inventorier les documents que la collection possède; et l' on attend des jeunes qui s' occupent de la publication du reste non sérieusement touché. Dans mes recherches sur tous les documents de toutes sortes que Grohmann avait prévu de publier dans sa Chrestomathie, qui a vu le jour, entièrement revue corrigée et élargie par moi-même dans deux volumes ${ }^{14}$, je suis tombé sur un certain nombre de papyrus, inventoriés par Grohmann et qu' il voulait publier, mais dont il n' a laissé que quelques notices et dont les originaux ont tout à fait disparu dans la collection de Heidelberg. Toutes mes longues recherches n'ont abouti jusqu'à maintenant à rien. Et pourtant les pièces de toute la collection étaient bien gardées, à l'intérieur de la Bibliothèque Universitaire, où elles sont restées jusqu'à la fondation de l' Institut de Papyrologie et de sa séparation des locaux bibliothécaires en 1976.

\footnotetext{
11 Khoury, Wahb ibn Munabbih 4 et Bibliographie.

12 Jahn, Vom frühislamischen 153-200.

13 Dietrich, Zum Drogenhandel.

14 Chrest.Khoury I et II.
} 
Peut-être que ces textes ont été déplacés, pour tomber dans la main d'un spécialiste, qui les garda à l'intérieur de sa propre bibliothèque, ou pour finir dans des caisses étrangères et ainsi appartenir à une autre institution ou tout simplement trouver leur fin dans un incendie ou une destruction quelconque. On ne peut le dire!

De quoi s' agit-il dans ces pièces qui comptent 159 pièces? Il est bon de donner quelques notions, au moins générales, pour ouvrir les yeux sur des pertes possibles, à l'intérieur de chaque collection à travers le monde, et ainsi avoir des éléments de compléments heureux:

$\mathrm{V} / \mathbf{1}$

D'abord nous avons d'eux des numéros d'inventaires. Ces numéros commencent assez bas, par le chiffre 63, pour aller de centaine en centaine et arriver jusquà 1451. Ils ne sont pas consécutifs, mais ont dû être pris de leurs anciens numéros, pour être travaillés, sans qu' ils aient appartenu à un même fond suivi, comme c'est le cas, assez souvent, au moins au début du classement de ces documents, après leur acquisition et les premiers essais de les rendre accessibles aux chercheurs.

Grohmann, qui semble avoir voulu les travailler, pour les publier, ne mentionne cependant pas toujours sur quel matériel chaque document a été écrit, et donc trouvé par lui dans la collection. Quelques fois il le mentionne, ce qui fait qu'il a dû disposer de certaines pièces de manière sûre; alors que concernant d'autres, presque la majorité, il n'y a aucune mention à ce sujet; ce qui laisse penser que les documents ont dû lui échapper, avant qu' il n' ait pu terminer son travail de description assez générale, sans avoir pu retenir des indications précises sur la plupart de ces textes. Quant au sigle général de PSR, employé par lui, il ne peut pas jeter de la lumière sur ce côté, car tous les textes de la collection portent cette étiquette, qui concernait et concerne toutes les pièces de toutes sortes, qui forment partie de la collection de Heidelberg dans l'ensemble. Donc là où le matériel porte une marque spéciale de Grohmann, comme «papyrus fin » ou «moyennement fin » etc., on peut conclure avec certitude qu'il s' agissait d'un texte sur papyrus; mais ces notations sont malheureusement très rares.

Les 159 textes n'étaient pas tous complets, mais certains, assez nombreux, étaient endommagés, à très endommagés:

Comme PSR 151v., qui forme une lettre d'affaires, avec la mention «sehr beschädigt » (très endommagé), alors que le recto de cette lettre est formée de 9 lignes, dont les deux premières sont vides, et que de 5 à 9 sont plus remplies. De même 181r, un contrat de bail (Pachtvertrag) de 11 lignes, avec des lacunes, du III ${ }^{\mathrm{e}}$ siècle, portant la date de $269 \mathrm{H}$. Un autre contrat de bail, PSR 69, de 12 
lignes, offre aussi des lacunes, mais est du III siècle, de même PSR 259, de 13 lignes, aussi avec des lacunes, de même PSR 259r, de 13 lignes.

D'autres étaient restés en bon état (gut erhalten), comme un contrat de mariage (répudiation / Trennung), formant 14 lignes. Ce contrat aurait été bien pour mon ancien élève Abdulbary, qui a publié une thèse de doctorat sur des contrats de mariage sur papyrus, avec une grande introduction sur la diplomatique, ou l'étude structurelle et idéelle des contrats au cours des premiers siècles en Egypte ${ }^{15}$. Un contrat de bail, qui est bien conservé en 16 lignes, est même daté de 209; quel dommage! Un autre PSR 1520 n'a que 8 lignes, sans autre indication.

Dans l' ensemble on a un certain nombre de contrats de bail qui étaient en bon état: ainsi PSR 63, avec un calcul du montant du bail et des dépenses, avec des données concernant les mesures du papyrus (décrit comme fin): $16.5 \times 12.4$; puis PSR 251, de 14 lignes, PSR 428, de 11 lignes. D'un autre PSR 116, de 9 lignes, il s' agirait vraisemblablement aussi d' un contrat de bail, qui serait, selon les indications des Grohmann, daté de $266 \mathrm{H}$.

Un grand nombre d' entre ces papyrus perdus (?) sont des listes d' impôts, sur lesquelles Faleh Hussein a aussi travaillé il y a vingt cinq ans, dans son doctorat sous ma direction ${ }^{16}$ :

Impôt capital (Kopfsteuer / jizya): PSR 1225, de 4 lignes. PSR 235V, avec une liste d'impôts de 12 lignes, sur un papyrus brun ouvert et moyennement fin, et aussi ses mesures que Grohmann a pu encore vérifier: $33.5 \times 17$. PSR 431v, avec un calcul des impôts, de 5 lignes. PSR $511 r$ des impôts de 9 lignes, et plus important PSR 531r, de même sur 30 lignes, ainsi que PSR 539 de 18 lignes, et du III ${ }^{\mathrm{e}}$ siècle.

Quelques listes de calculs (d'impôts) vont de même très haut dans le nombre

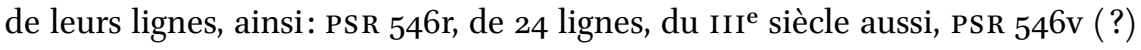
une liste de calculs de céréales, sur 29 lignes. D' autres nombreuses quittances de plusieurs lignes, comme PSR 615r, 16 lignes; PSR 618, 13 lignes, du III ${ }^{\mathrm{e}}$ siècle; PSR 622, 8 lignes, aussi du III ${ }^{\mathrm{e}}$ siècle, ainsi du même siècle, PSR 627, 12 lignes. Des listes de calcul d'impôts (vraisemblablement) sont assez nombreuses, comme PSR 1151, de 14 lignes, PSR 1157r, 10 lignes, PSR 1159 de 11 lignes.

Il est toujours très regrettable d'être confronté à des données pareilles, sans avoir la possibilité de voir un original, ou une copie de lui, pour pourvoir travailler, publier ou au moins dire quelque chose de plus sur le contenu et les modalités de la conclusion du contrat, ou au moins sur quelques détails

15 al-Mudarris 2009.

16 Hussein, Papyrologische 19-254 / 639-868. 
importants de cette conclusion. On voit dans l' ensemble qu' il s' agit là-dedans des données suivantes, que ces papyrus ont voulu attester par écrit:

- Des contrats de bail, dont plusieurs étaient assez complets, d' autres avec des lacunes, et même parfois avec certaines lignes vides, parce que non employées.

- Des listes d'impôts, très nombreuses, avec des pièces plus complètes, d' autres moins complètes, et d' autres avec de grosses lacunes.

- Des listes de calcul de toutes sortes, et pas seulement concernant des problèmes de taxation.

- Des listes de dépenses.

- Une liste d'ustensiles ménagers.

- Une liste de marchandises avec leurs prix.

- Une liste de biens.

- Des quittances de toutes sortes.

- Des listes de noms (affaires, impôts?).

- Un papyrus même concerne la vente d' une maison (PSR 1451, de 27 lignes), un autre l' achat d'une maison (PSR 190 plus 191, 33 lignes / 12 plus 21, de 270 H.).

- Et certaines lettres privées, avec règlements de certaines affaires.

- Même un contrat de mariage, bien conservé, PSR 209, 14 lignes, qu' on a vu plus haut.

Dans cette collection il y a une omoplate d'une chèvre, publiée (PSR 1204, $8 \times 17.5$, du I ${ }^{\text {er }} / \mathrm{II}^{\mathrm{e}}$ siècle $\mathrm{H}$. Le texte a été publié par Becker $)^{17}$. De même une lettre à un gouverneur, PSR 594r, de 9 lignes, avec des lacunes. Deux textes de protocoles, PSR 433r, 11 lignes, et 433v de 10 lignes, déjà publiés par Grohmann, dans ses textes de protocoles ${ }^{18}$.

Voilà un aperçu général de ces textes sur papyrus de la collection de Heidelberg. Je l'ai donné ici, en vue d'informer un public, intéressé à la papyrologie arabe surtout, et pour attirer l' attention sur d' autres textes possibles, qui pourraient avoir une relation quelconque avec de telles données. Il y a eu quelques papyrus anciens, qui seraient un complément à des pièces plus nombreuses de Heidelberg, qui ont fini par se retrouver en Amérique, dans la collection de

17 Dans P.Heid.Arab. I 7. Y. Rāgib en a publié deux lettres aussi, voyez plus haut, note 3, et W. Diem des remarques sur les publications en général Philologisches, 251-275; le même, Der Gouverneur 104-111.

18 CPR III/1 et aussi dans Chrest.Khoury I. 
Chicago: ainsi un début de papyrus sur la création du monde, qui rappelle le genre de récits attribués à Wahb Ibn Munabbih, comme le livre de Bad'al-khalq wa-qișaș al-anbiyā', d'Abū Rifā'a 'Umāra Ibn Wathīma Ibn Mūsā al-Fārisī, que j' ai publiés en $1978^{19}$, et qui remontent à Wahb en premier lieu; l'un d' eux a été publié par Nabia Abbott dans ses «Studies $»^{20}$.

Pour le reste je n' ai pas besoin de répéter qu' il faut à tout prix faire connaître tout ce qui n'est pas connu de chaque collection papyrologique, car il y a toujours des textes qui viennent des mêmes fouilles, des mêmes lieux, et qui offrent des ressemblances étonnantes, qu' on peut faire connaître, à la lumière d'autres qui sont connus, publiés et donc devenus accessibles. Ceci pourrait aider à replacer ce qui a été perdu, à sa juste place, et peut-être lui trouver un complément adéquat. De plus travailler des papyrus, n'est pas une affaire simple à accomplir; et il faut être prudent, honnête, et savoir jusqu' où vont ses propres compétences, afin de savoir rendre le service attendu, et aider par là la science concernant les époques islamiques anciennes à donner un bon rendement, et non une idée faussée de la réalité historique, linguistique et sociale de ce passé, malheureusement toujours difficile à cerner, à analyser de manière entièrement satisfaisante. De bonnes publications papyrologiques seraient le meilleur moyen d'arriver à des résultats plus réels et donc plus positifs.

Il faut insister, pour terminer, sur le fait que les spécialistes doivent s' entraider, s'épauler, pour arriver au meilleur résultat possible, pour le bien de notre recherche scientifique et l'intérêt des pays en question: les documents sur papyrus, en effet, ne mentent pas et ne faussent donc pas la réalité historique, que d'autres textes ne reproduisent pas toujours avec fidélité. Là - dessus le travail de Abdelbary al-Mudarris est particulièrement intéressant, surtout concernant le rôle positif joué par la femme musulmane dans les documents de mariage, sur lequel il faut attirer l'attention, de manière spéciale aujourd'hui ${ }^{21}$. De plus Y. Rāgib a publié une étude générale sur les «Actes de vente d'esclaves et d'animaux d'Egypte médiévale », comme suite à son premier volume sur ce sujet ${ }^{22}$ : Là - dedans il analyse systématiquement les «caractères externes » et «internes » de ces actes, y compris l' objet, les questions de prix et des autres modalités touchant toute sorte de clauses et de témoignages. Ces données bibliographiques n'ont par ailleurs aucune pré-

\footnotetext{
19 Khoury, Les légendes.

20 Abbott, Studies.

21 al-Mudarris, Papyrologische.

22 Rāgib, Actes de vente 1 et 2.
} 
tention d'être exhaustives. Des renseignments beaucoup plus complets sont amplement amenés dans le Arabic Papyrology Database ${ }^{23}$.

\section{Bibliographie}

Abbott, N., Studies in Arabic literary papyri ( (Historical texts [OIP 75]), Chicago 1957.

Dietrich, D., Zum Drogenhandel im islamischen Ägypten. Eine Studie über die arabische Handschrift 912 der Heidelberger Papyrussammlung, Heidelberg 1954.

. Die arabischen Papyri des Topkapı Sarayi-Museums in Istanbul, in Der Islam 33 (1957), 37-50.

Diem, W., Philologisches zu den arabischen Aphrodito - Papyri, in Der Islam 61 (1984), 251-275.

. Der Gouverneur an den Pagarchen. Ein verkannter Papyrus vom Jahre 65 der Hiğra, in Der Islam 60 (1983), 104-111.

Hinds, M., al-Maghāzī, in Encyclopaedia of Islam. New Edition, 5: 1161.

Hussein, F., Das Steuersystem in Ägypten von der arabischen Eroberung bis zur Machtergreifung der Ṭulüniden 19-254 / 639-868. Mit besonderer Berücksichtigung der Papyrusurkunden, Francfort a. M. 1982.

al-Jābirī, M., Takwīn al-'aql al-'arabī, $2^{\mathrm{e}}$ éd., Beyrouth 1985.

Jahn, K., Vom frühislamischen Briefwesen, in Archív Orientální 9 (1937), 153-200.

Jāsir Abū Safiyya, Bardìyyāt Qurra Ibn Sharīkal-Absī. Dirāsa wa-tahqū̄q, Riyad 1425/2004. Khoury, R.G., L' apport spécialement important de la papyrologie dans la transmission et la codification des plus anciennes versions des Mille et Une Nuits et d'autres livres des deux premiers siècles islamiques, in P.M. Sijpesteijn et L. Sundelin (eds.), Papyrology and the history of early Islamic Egypt (Islamic history and civilization. Studies and texts vol. 55), Leyde 2004, 63-95.

'Abd Alläh Ibn Lahī'a. Juge et grand maître de l'Ecole Egyptienne. Avec éd. Critique de l'unique rouleau de papyrus arabe conservéà Heidelberg (Codices Arabici Antiqui IV), Wiesbaden 1986.

- Les légendes prophétiques dans l'Islam depuis le I ${ }^{e r}$ jusqu'au III siècle de l'Hégire. (Codices Arabici Antiqui III), Wiesbaden 1978.

—. Asad ibn Mūsā. K. az-Zuhd. Nouvelle éd. Revue, corrigée et augmentée de tous les certificats de lecture (Codices Arabici Antiqui II), Wiesbaden 1976, 91-108.

. Wahb ibn Munabbih (Codices Arabici Antiqui I), Wiesbaden 1972.

Mackay, P.A., Certificates of transmission on a manuscript of the Maqāmāt of Harīrì (Ms. Cairo, Adah 105) (Transactions of the American Philosophic Society, N.s. 61/4), Philadelphia 1971.

23 http://orientw.uzh.ch:808o/apd/project.jsp. 
Mélamède, G., The meetings at al-'Aqaba, Le Monde Oriental 28 (1934), 17-58.

al-Mudarris, A., Papyrologische Untersuchungen zur arabischen Diplomatik anhand von Eheurkunden (Codices Arabici Antiqui X), Wiesbaden 2009.

Rāgib, Y., Actes de vente d'esclaves et d'animaux d'Egypte médiévale 2 (Cahier des Annales Islamologiques 28), Le Caire 2006.

Actes de vente d'esclaves et d'animaux d'Egypte médiévale 1 (Cahier des Annales Islamologiques 23), Le Caire 2002.

Lettres nouvelles de Qurra ibn Šarīk, in Journal of Near Eastern Studies 40 (1981), 173-187.

Seider, R., Aus der Arbeit der Universitätsinstitute. Die Universitätspapyrussammlung, in HeidelbergerJahr - Bücher 8 (1964), 142-203.

Sellheim, R., Gelehrte und Gelehrsamkeit im Reiche der Chalifen, in H. Strasburger (ed.), Festschrift für P. Kirn, Berlin 1963, 54-79.

Sijpesteijn, P.M., Une nouvelle lettre de Qurra ibn Šarikk. P.Sorb. inv. 2345, in Annales Islamologiques 45 (2011), 257-267. . et L. Sundelin (eds.), Papyrology and the history of early Islamic Egypt (Islamic history and civilization. Studies and texts vol. 55), Leyde 2004.

Tarābīshī, G., Ishkāliyyāàt al-'aql al-'arabī, Beyrouth et Londres 1998.

Vajda, G., Les certificats de transmission dans les manuscrits arabes de la Bibliothèque Nationale de Paris, Paris 1956. 